\title{
Configuration spaces on the sphere and higher loop spaces
}

\author{
Paolo Salvatore
}

\begin{abstract}
We show that the homology over a field of the space $\Lambda^{n} \Sigma^{n} X$ of free maps from the $n$-sphere to the $n$-fold suspension of $X$ depends only on the cohomology algebra of $X$ and compute it explicitly. We compute also the homology of the closely related labelled configuration space $C\left(S^{n}, X\right)$ on the $n$-sphere with labels in $X$ and of its completion, that depends only on the homology of $X$. In many but not all cases the homology of $C\left(S^{n}, X\right)$ coincides with the homology of $\Lambda^{n} \Sigma^{n} X$. In particular we obtain the homology of the unordered configuration spaces on a sphere.
\end{abstract}

MSC (2000): 55P48, 55R80, 55S12.

Keywords: Configuration space, loop space, homology operation.

\section{Introduction}

In order to compute the homology of the space of based maps $\Omega^{n}(Y)=\operatorname{map}_{*}\left(S^{n}, Y\right)$ from the $n$ sphere to a CW-complex $Y$, in general one needs to know a great deal of information on $Y$. However, when $Y=\Sigma^{n} X$ is a $n$-fold suspension, a classical result by Milgram 9] states that the homology of $\Omega^{n} \sum^{n} X$ depends just on the homology of $X$. An explicit description is given in 3 . This depends upon the existence of a small model for the mapping space. Namely the configuration space $C\left(\mathbb{R}^{n}, X\right)$ of pairwise distinct points in $\mathbb{R}^{n}$ with labels in $X$, modulo base point cancellation, is homotopy equivalent to $\Omega^{n} \Sigma^{n} X$ when $X$ is connected [6]. In general $\Omega^{n} \Sigma^{n} X$ is the group completion of $C\left(\mathbb{R}^{n}, X\right)$.

Let us turn our attention to the free mapping space $\Lambda^{n} Y=\operatorname{map}\left(S^{n}, Y\right)$. In the case $n=1$ the homology of $\Lambda \Sigma X$ depends only on the homology of $X$ [8]. This depends again on the existence of a small model for the mapping space, the configuration space $C\left(S^{1}, X\right)$ of points in the circle with labels in $X$ [2], for $X$ connected. This does not extend to all $n$. For $X$ connected the configuration space $C\left(S^{n}, X\right)$ is homotopy equivalent to the section space of a bundle over $S^{n}$ with fiber $\Sigma^{n} X$ [2], obtained from the tangent bundle of the sphere $\tau_{n}$ by adding a point at infinity to each fiber and smashing it with $X$. In general this section space is a kind of completion of $C\left(S^{n}, X\right)$. When $n \neq 1,3,7, S^{n}$ is not parallelizable, so that one does not expect $C\left(S^{n}, X\right)$ to be homotopy equivalent to the free mapping space $\Lambda^{n} \Sigma^{n} X$.

In this paper we compare the homotopy types of these spaces and we compute their homology with coefficients in any field. Up to homotopy there are fibrations $C\left(\mathbb{R}^{n}, X\right) \rightarrow C\left(S^{n}, X\right) \rightarrow \Sigma^{n} X$ (for $X$ connected) and $\Omega^{n} \Sigma^{n} X \rightarrow \Lambda^{n} \Sigma^{n} X \rightarrow \Sigma^{n} X$, induced by the evaluation at one point. Since the basis of both fibrations is a suspension, we can reconstruct the total spaces by means of the clutching functions $\Sigma^{n-1} X \times \Omega^{n} \Sigma^{n} X \rightarrow \Omega^{n} \Sigma^{n} X$. It turns out that the clutching function of the first fibration comes from the action of the little $n$-discs operad $S^{n-1} \times X \times C\left(\mathbb{R}^{n}, X\right) \rightarrow C\left(\mathbb{R}^{n}, X\right)$. This action adds to a 'cloud' of labelled points in $C\left(\mathbb{R}^{n}, X\right)$ an extra point in the direction parametrized by $S^{n-1}$ with label parametrized by $X$. The same argument works when $X$ is not connected. In general we get decompositions both for $C\left(S^{n}, X\right)$ (Theorem 11) and its completion (Theorem [6), that coincide for $X$ connected. The decomposition is compatible with the Snaith splitting (Proposition [5). The clutching function induces in homology the so called Browder operation, that has been computed explicitly for $C\left(\mathbb{R}^{n}, X\right)$ in 3 . For example in characteristic 0 and for $n=2$ we get the adjoint action of the homology of $X$ on the free Gerstenhaber algebra that it generates. This allows to determine the homology of $C\left(S^{n}, X\right)$, that depends only on the homology of $X$ (Corollary 3). In particular for $X=S^{0}$ we obtain the homology of the unordered configuration spaces on $S^{n}$. We write down an 
explicit basis of their homology in Theorem [18 The case when $n$ is even and the characteristic is odd is not covered by the methods in [1.

Let us consider now the evaluation fibration of the mapping space $\Lambda^{n} \Sigma^{n} X$. In this case the clutching function is obtained by twisting the first argument $S^{n-1} \times X \rightarrow \Omega^{n} \Sigma^{n} X$ and then applying as before the little $n$-discs action $S^{n-1} \times\left(\Omega^{n} \sum^{n} X\right)^{2} \rightarrow \Omega^{n} \sum^{n} X$ (Theorem 11) . The twist is the composition operation induced by the element $c \in \pi_{n-1}\left(\Omega_{1}^{n} S^{n}\right)$ adjoint to the Whitehead product of the generator $\pi_{n}\left(S^{n}\right)$ with itself. This happens exactly because $c$ classifies the fiberwise compactification of the tangent bundle of the $n$-sphere (Lemma 10).

Now $c$ goes in homology (up to shift of component) to the Browder operation of the identity $\iota \in H_{0}\left(\Omega^{n} S^{n}\right)$ with itself. This implies that the clutching function of the mapping space in homology depends only on the cohomology algebra of $X$ (for finite type), and so does the homology of $\Lambda^{n} \Sigma^{n} X$ (Corollary 12). We show in Example [16 that the homology of $\Lambda^{n} \sum^{n} X$ does not depend just on the additive homology of $X$. As an application we write down an explicit basis of the homology of the mapping space $\Lambda^{n} S^{m}$, for $n \leq m$, in Theorems 18 and 19

Let us compare the configuration space (or its completion for $X$ not connected) and the mapping space: when $c$ vanishes they are homotopy equivalent. This happens when either $X$ is a suspension, or $n=1,3,7$, or for $n$ odd after inverting the prime 2 (Proposition 9). Moreover the two spaces have the same homology either mod 2 (Corollary 13), or for $X$ connected and rationally (Corollary 14). In general they do not have the same homology for $n$ even at odd primes (Example 16), or rationally when $X$ is not connected (Example 15).

We present also a homotopy pullback decomposition of our spaces, induced by decomposing the $n$-sphere of the domain as union of two discs (Propositions 20] and 21). In this case the twist occurs in the diagram describing the configuration space. We apply the induced sequence of homotopy groups to show in Example 22 that the configuration space and the mapping space are not rationally homotopic for $n=2$ and $X=\mathbb{C P}^{2}$, but they have the same homology over all fields.

It would be interesting to phrase our computations in terms of the higher Hochschild homology spectral sequence described in 5.9 of [10].

In sections 112 and 3 we consider respectively labelled configuration spaces on the sphere, the associated spaces of sections, and mapping spaces. In section 4 we compute some examples and we compare the homology of the spaces above. In section [5] we present the homotopy pullback decomposition and an application.

I am grateful to Sadok Kallel for many helpful conversations.

\section{Configuration spaces}

Let $X$ be a based CW-complex, not necessarily connected. Let $n$ be a positive integer.

We recall some definitions from 2 . Let $F_{k}(M)$ be the space of pairwise distinct $k$-tuples in a manifold $M$. We consider the elements of the space $F_{k}(M) \times_{\Sigma_{k}} X^{k}$ as finite sets of points in $M$ with a label in $X$. The configuration space $C(M, X)$ is the quotient of the disjoint union $\coprod_{k} F_{k}(M) \times_{\Sigma_{k}} X^{k}$ under the equivalence relation induced by cancelling points labelled by the base point of $X$. Given a closed subset $A \subset M$, the relative configuration space $C(M, A ; X)$ is the quotient induced by cancelling all points located in $A$ and those labelled by the base point.

We identify $S^{n}=\mathbb{R}^{n} \cup \infty$, based at infinity. Let $D_{n} \subset \mathbb{R}^{n}$ be the unit disc. The sequence $D_{n} \rightarrow$ $S^{n} \rightarrow\left(S^{n}, D_{n}\right)$, for $X$ connected, induces a quasifibration $C\left(D_{n}, X\right) \rightarrow C\left(S^{n}, X\right) \rightarrow C\left(S^{n}, D_{n} ; X\right)$ (Lemma p.178 in [2]). Now $\Sigma^{n} X \subset C\left(S^{n}, D_{n} ; X\right)$ is a strong deformation retract, seen as the subspace of configurations with a single labelled point [7]. We will construct a homotopy pushout decomposition of $C\left(S^{n}, X\right)$, by pulling back to the total space the decomposition of $\Sigma^{n} X=S^{n} \wedge X$ induced by splitting $S^{n}$ as union of the $n$-discs $D_{n}$ and $E_{n}=\overline{S^{n}-D_{n}}$. The quasifibration over $D_{n+} \wedge X$ and $E_{n} \wedge X$ is homotopically trivial. It remains to glue the two pieces together. This idea works even when $X$ is not connected. 
We recall that $C\left(D_{n}, X\right) \simeq C\left(\mathbb{R}^{n}, X\right)$ is homotopy equivalent to the free algebra on the operad of little $n$-discs generated by $X$. In particular there is an action of the space of two little $n$-discs $\mathcal{D}_{n}(2)$ by a map $\rho_{2}: \mathcal{D}_{n}(2) \times C\left(\mathbb{R}^{n}, X\right) \times C\left(\mathbb{R}^{n}, X\right) \rightarrow C\left(\mathbb{R}^{n}, X\right)$. Let us restrict this map to the deformation retract $S^{n-1} \subset \mathcal{D}_{n}(2)$ in the first factor and to $X \subset C\left(\mathbb{R}^{n}, X\right)$, the subspace of configurations consisting of a single labelled point located at the origin, in the second factor. We get a map $\lambda: S_{+}^{n-1} \wedge X \times C\left(\mathbb{R}^{n}, X\right) \rightarrow C\left(\mathbb{R}^{n}, X\right)$, that we shall call the Browder map. Up to deformation $\lambda(z, x, c)$ is the configuration obtained from the configuration $c$ by adding a point outside $c$ in direction $z$ with label $x$.

Theorem 1. There is a homotopy pushout

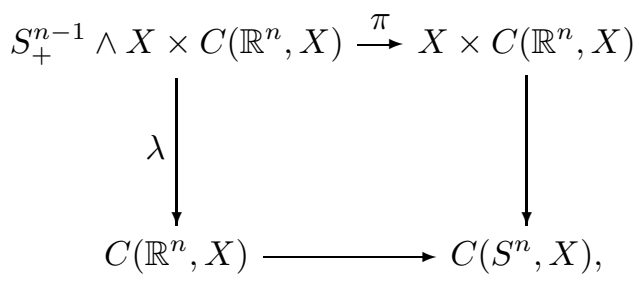

where $\lambda$ is the Browder map and $\pi$ the projection.

Proof. Let us consider the closed deformation retract $K$ of $C\left(S^{n}, X\right)$ containing the configurations with at most one labelled point located outside $D_{n}$. The deformation is obtained by pushing the particles away from the north pole and deforming slightly the labels towards the base point. Let $A, B \subset K$ be the closed subsets such that the point located outside $D_{n}$, if it exists, is respectively in $2 D_{n}$ and $2 E_{n}$. Clearly $A \cup B=K$. Moreover the inclusion $C\left(D_{n}, X\right) \subset A$ is a homotopy equivalence. On the other hand $A \cap B \cong S_{+}^{n-1} \wedge X \times C\left(D_{n}, X\right)$, and $B \cong D_{n+} \wedge X \times C\left(D_{n}, X\right)$. With these identifications the map $A \cap B \rightarrow B$ is induced by the inclusion $S^{n-1} \subset D_{n}$. Moreover the inclusion $A \cap B \rightarrow A$ is homotopic to the Browder map.

From now on we consider homology with coefficients in a field $K$. We recall the definition of the Browder operation: let $Y$ be a space acted on by the little $n$-discs operad. Let $e_{n-1} \in H_{n-1}\left(\mathcal{D}_{n}(2)\right)$ be the class represented by the deformation retract $S^{n-1} \subset \mathcal{D}_{n}(2)$. Then the Browder operation, for $x \in H_{p}(Y)$ and $y \in H_{q}(Y)$, is defined by $[x, y]:=\rho_{2 *}\left(e_{n-1} \otimes x \otimes y\right) \in H_{p+q+n-1}(Y)$. This differs from (5.7) of $\left[3\right.$ by the sign $(-1)^{(n-1) p+1}$.

For $n=1$ the fundamental class of $S^{0}$ is $\{1\}-\{-1\}$, so that the Browder operation is the commutator of the Pontrjagin product.

Definition 2. The Browder action is the homomorphism

$$
[,]=\lambda_{*}: \Sigma^{n-1} \tilde{H}(X) \otimes H\left(C\left(\mathbb{R}^{n}, X\right)\right) \rightarrow H\left(C\left(\mathbb{R}^{n}, X\right)\right) .
$$

Recall from Theorem 3.1 in [3] that the homology $\tilde{H}\left(C\left(\mathbb{R}^{n}, X\right)\right)$ depends functorially on the homology $\tilde{H}(X)$. For example in characteristic 0 and for $n>1$ the former is the free $n$-algebra on the latter. A $n$-algebra is a graded variation of a Poisson algebra [11. In our case the Pontrjagin product is the commutative product and the Browder operation is the bracket (up to sign). The Browder action for $\operatorname{char}(K)=0$ is exactly the adjoint action of the space of generators $\tilde{H}(X)$ on $H\left(C\left(\mathbb{R}^{n}, X\right)\right)$.

In positive characteristic there are additional homology operations generating $H\left(C\left(\mathbb{R}^{n}, X\right)\right)$ over $H(X)$, the unstable Dyer-Lashof operations. See (1.1),(1.3) of [3] and Proposition 17

In general the Browder action can be computed explicitly and depends just on the homology of $X$ by Thm. $1.2(5,6,8)$ and Thm. 1.3 (4) of [3. For example mod 2 the action is trivial on all Dyer-Lashof operations except the top one. Recall that $H(C(\mathbb{R}, X))$ is the free associative algebra on $\tilde{H}(X)$.

The Mayer-Vietoris sequence implies the following corollary. 
Corollary 3. The homology of $C\left(S^{n}, X\right)$ depends only on the homology of $X$ and is the direct sum of the suspended kernel and the cokernel of the Browder action on the homology of $C\left(\mathbb{R}^{n}, X\right)$.

The configuration spaces $C(M, X)$, for a manifold $M$, are naturally filtered by subspaces $C_{k}(M, X)$, having at most $k$ particles. We denote the subquotients by $D_{k}(M, X)=C_{k}(M, X) / C_{k-1}(M, X)$ and $D_{k}^{n}(X)=D_{k}\left(\mathbb{R}^{n}, X\right)$. For example the space $D_{k}\left(S^{n}, S^{0}\right)$ is the unordered configuration space on the sphere $C_{k}\left(S^{n}\right)=F_{k}\left(S^{n}\right) / \Sigma_{k}$.

The homology $H(C(M, X))$ splits naturally as sum of $H\left(D_{k}(M, X)\right)$, as $k$ varies. Compare Lemma 4.2 p.238 in [3. This explains the first assertion of Corollary [3 as

$H\left(C\left(S^{n}, X\right)\right) \cong \bigoplus_{k} H\left(F_{k}\left(S^{n}\right) ; \tilde{H}(X)^{\otimes k}\right)$.

The Browder map is compatible with the filtration by number of particles, so that we have the following refinement:

Corollary 4. The reduced homology of $D_{k}\left(S^{n}, X\right)$, for $k>1$, is the direct sum of the suspended kernel and the cokernel of the Browder action $\Sigma^{n-1} \tilde{H}(X) \otimes \tilde{H}\left(D_{k-1}^{n}(X)\right) \rightarrow \tilde{H}\left(D_{k}^{n}(X)\right)$.

There is a geometric version of this corollary. Recall that $C(M, X)$ is stably homotopy equivalent to the wedge sum of $D_{k}(M, X)$, indexed over $k$ [2]. The Browder map defines a stable map $b_{k}$ : $\Sigma^{n-1} X \wedge D_{k-1}(X) \rightarrow D_{k}(X)$, by restriction to the relevant wedge summands. For example the map $b_{2}$ is the restriction to the stable summand $S^{n-1} \wedge X \wedge X$ of the quotient map $S_{+}^{n-1} \wedge X \wedge X \rightarrow$ $S_{+}^{n-1} \wedge_{\mathbb{Z}_{2}}(X \wedge X)$.

Proposition 5. The $k$-th summand of the stable splitting $D_{k}\left(S^{n}, X\right)$ is stably homotopy equivalent to the cofiber of $b_{k}: \Sigma^{n-1} X \wedge D_{k-1}^{n}(X) \rightarrow D_{k}^{n}(X)$ for $k>1$ and to $\Sigma^{n} X \vee X$ for $k=1$.

Proof. We obtain a homotopy pushout ( $k$ ) expressing the homotopy type of $C_{k}\left(S^{n}, X\right)$, if we replace, in the diagram of Theorem $1, C$ by $C_{k-1}$ in the top row and by $C_{k}$ in the bottom row. For $k=1$ this proves the proposition. For $k>1$ the cofiber diagram of $(k-1)$ and $(k)$ gives $D_{k}\left(S^{n}, X\right)$ as homotopy pushout. The top map $\pi_{k}$ of such diagram is a stable retraction, and $b_{k}$ is exactly the restriction of the left hand side map $\lambda_{k}$ to the stable kernel of $\pi_{k}$.

\section{Section spaces}

We recall that there is a 'scanning' map $C\left(D_{n}, X\right) \rightarrow \Omega^{n} \Sigma^{n} X \cong \operatorname{map}_{*}\left(D_{n} / S^{n-1}, D_{n} / S^{n-1} \wedge X\right)$. Let $i: X \rightarrow \Omega^{n} \Sigma^{n} X$ be the adjoint of the identity. Roughly speaking, if we scan a configuration, we obtain a $n$-fold loop, sending small discs centered at the configuration points to $\Sigma^{n} X \cong D_{n} / S^{n-1} \wedge X$, by identifying them to the unit disc and applying $i$ to the label of the center. The $n$-fold loop sends everything else to the base point.

The scanning map can be extended to $C\left(S^{n}, X\right)$ by using the exponential map of the sphere. Let $\Sigma^{n} X \rightarrow \tau_{n}^{+}(X) \rightarrow S^{n}$ be the bundle obtained by smashing $X$ fiberwise with the fiberwise one-point compactification $\tau_{n}^{+}$of the tangent bundle $\tau_{n}$ of the $n$-sphere. Then the scanning map goes from $C\left(S^{n}, X\right)$ to the space $\Gamma\left(\tau_{n}^{+}(X)\right)$ of sections of the bundle. Up to homotopy the scanning maps fit into a diagram from the sequence

$$
C\left(D_{n}, X\right) \rightarrow C\left(S^{n}, X\right) \rightarrow C\left(S^{n}, D_{n} ; X\right)
$$

to the evaluation fibration

$$
\Omega^{n} \Sigma^{n} X \rightarrow \Gamma\left(\tau_{n}^{+}(X)\right) \stackrel{e v}{\longrightarrow} \Sigma^{n} X .
$$

The scanning maps $C\left(D_{n}, X\right) \rightarrow \Omega^{n} \Sigma^{n} X$ and $C\left(S^{n}, X\right) \rightarrow \Gamma\left(\tau_{n}^{+}(X)\right)$ are homotopy equivalences if and only if $X$ is connected.

We can define the Browder map for the $n$-fold loop space $\Omega^{n} \Sigma^{n} X$, similarly as for the configuration space, by using the action $\mathcal{D}_{n}(2) \times \Omega^{n} \Sigma^{n} X \times \Omega^{n} \Sigma^{n} X \rightarrow \Omega^{n} \Sigma^{n} X$ of the $n$-discs operad, and restricting the action in the second factor to the subspace $X \stackrel{i}{\longrightarrow} \Omega^{n} \Sigma^{n} X$. 
If $X$ is connected then the next theorem reduces to Theorem 1 Also the proof is an adaptation of the proof of Theorem 1 to the general case.

Theorem 6. There is a homotopy pushout

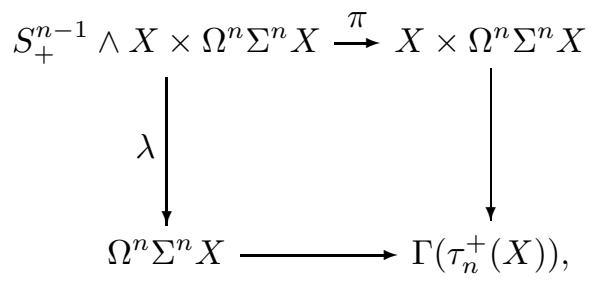

where $\lambda$ is the Browder map and $\pi$ the projection.

Proof. Let $e v: \Gamma\left(\tau_{n}^{+}(X)\right) \rightarrow \tau_{n}(N)^{+} \wedge X \cong \Sigma^{n} X$ be the fibration evaluating at the north pole $N$.

We construct a map $(\alpha, \beta, \gamma)$ from the diagram

$$
D_{n+} \wedge X \times \Omega^{n} \Sigma^{n} X \longleftarrow S_{+}^{n-1} \wedge X \times \Omega^{n} \Sigma^{n} X \longrightarrow M(\lambda),
$$

where $M(\lambda)$ is the mapping cylinder of the Browder map, to the diagram

$$
e v^{-1}\left(D_{n+} \wedge X\right) \longleftarrow e v^{-1}\left(S_{+}^{n-1} \wedge X\right) \longrightarrow e v^{-1}\left(E_{n} \wedge X\right) \text {. }
$$

We construct $\alpha$ so that it covers an automorphism of $D_{n+} \wedge X$ homotopic to $-1 \wedge X$. We identify both the southern and the northern hemisphere to the unit disc, by projection from the opposite pole. This gives a trivialization of the tangent bundle on each hemisphere.

The map $\alpha(z \wedge x, y) \in \Gamma\left(\tau_{n}^{+}(X)\right)$ is defined by $y \in M a p\left(D_{n}, \partial D_{n} ; \Sigma^{n} X\right)$ on the southern hemisphere. Let $\phi:\left(D_{n}, S^{n-1}\right) \rightarrow\left(S^{n}, \infty\right)$ be the relative homeomorphism $\phi(x)=x /(1-|x|)$. In the northern hemisphere we have the adjoint $n$-fold loop $\alpha(z \wedge x, y)(w)=\phi(2(w-z / 4)) \wedge x$ if $|w-z / 4| \leq 1 / 2$, and otherwise $w$ goes to the point at infinity.

Similarly, on the cylinder $I \times S_{+}^{n-1} \wedge X \times \Omega^{n} \Sigma^{n} X \subset M(\lambda), \gamma(t, z \wedge x, y)$ is defined in the southern hemisphere by $y$. On the northern hemisphere $\gamma(t, z \wedge x, y)(w)=\phi(2(w-z(t+1) / 4)) \wedge x$ for $|w-z(t+1) / 4| \leq 1 / 2$ and otherwise $w$ goes to the point at infinity. The map $\gamma$ sends the end of the mapping cylinder $\Omega^{n} \Sigma^{n} X$ to $e v^{-1}(*)$ by projection from the north pole.

Both $\alpha$ and $\beta$ are homotopy equivalences, because they cover an automorphism of the basis and induce homotopy equivalences of the fibers over points of all components. Finally $\gamma$ is a homotopy equivalence, since the domain has $\Omega^{n} \Sigma^{n} X$ as deformation retract, identified by $\gamma$ to $e v^{-1}(*) \simeq$ $e v^{-1}\left(E_{n} \wedge X\right)$. By the homotopy invariance of homotopy pushouts the theorem is proved.

Also in this case we call Browder action the homomorphism $\lambda_{*}: \Sigma^{n-1} \tilde{H}(X) \otimes H\left(\Omega^{n} \Sigma^{n} X\right) \rightarrow$ $H\left(\Omega^{n} \Sigma^{n} X\right)$ induced by the Browder map.

Corollary 7. The homology of $\Gamma\left(\tau_{n}^{+}(X)\right)$ depends only on the homology of $X$, and is the direct sum of the suspended kernel and the cokernel of the Browder action on the homology of $\Omega^{n} \Sigma^{n} X$.

Proof. Recall that $H\left(\Omega^{n} \Sigma^{n} X\right)$ is obtained from $H\left(C\left(\mathbb{R}^{n}, X\right)\right)$, according to the group completion theorem, by adding the inverses of the components.

In the case $n>1 \pi_{0}\left(C\left(\mathbb{R}^{n}, X\right)\right)=\mathbb{N}\left[\pi_{0}(X)\right]$, and $\pi_{0}\left(\Omega^{n} \Sigma^{n} X\right)=\mathbb{Z}\left[\pi_{0}(X)\right]$. We must only compute the Browder action on the inverse of a component $a \in \pi_{0}(X) \subset H\left(\Omega^{n} \Sigma^{n} X\right)$. But by the Poisson relation and since the Browder action on the unit is trivial, we obtain $\left[x, a^{-1}\right]=-[x, a] a^{-2}$.

In the case $n=1 \pi_{0}(C(\mathbb{R}, X))$ and $\pi_{0}(\Omega \Sigma X)$ are respectively the free monoid and the free group on the based set $\pi_{0}(X)$, and the Pontrjagin product is clear.

Example 8. If $X=S^{0} \vee S^{0}$, then $\Gamma\left(\tau_{n}^{+}(X)\right)$ is homotopy equivalent to the section space of the bundle $F_{3}\left(\mathbb{R}^{n+1}\right) \rightarrow F_{2}\left(\mathbb{R}^{n+1}\right)$ defined by forgetting the third point of a configuration [5]. 


\section{$3 \quad$ Higher loop spaces}

In some special cases the section space and the mapping space coincide.

Proposition 9. The fibration $\Sigma^{n} X \rightarrow \tau_{n}^{+}(X) \rightarrow S^{n}$ is trivial, so that the section space is homotopic to the ordinary mapping space $\Lambda^{n} \Sigma^{n} X$, in the following cases:

1. When $X$ is a suspension $X=\Sigma Y$;

2. For $n=1,3,7$;

3. For $n$ odd and away from the prime 2.

Proof. 1) The tangent bundle of the sphere is trivialized by adding a trivial line bundle. Thus $\tau_{n}^{+}(\Sigma Y) \cong\left(\tau_{n} \oplus \mathbb{R}\right)^{+}(Y) \cong \Sigma^{n+1} Y \times S^{n}$.

2) The spheres $S^{1}, S^{3}, S^{7}$ are parallelizable.

3) Follows from the following lemma, since $2\left[\iota_{n}, \iota_{n}\right]=0 \in \pi_{2 n-1}\left(S^{n}\right)$ for $n$ odd, where $\iota_{n} \in \pi_{n}\left(S^{n}\right)$ is the generator and the brackets denote the Whitehead product.

Lemma 10. The fibration $S^{n} \rightarrow \tau_{n}^{+} \rightarrow S^{n}$ with section $S^{n} \rightarrow \tau_{n}^{+}$at infinity is classified by the adjoint of the Whitehead product $\left[\iota_{n}, \iota_{n}\right]$ in $\pi_{n-1}\left(\Omega_{1}^{n} S^{n}\right)$.

Proof. The bundle $\tau_{n}^{+}$is trivial if we forget the section, because it is the sphere bundle of $\tau_{n} \oplus \mathbb{R}$, and its section can be identified to the diagonal $\Delta: S^{n} \rightarrow S^{n} \times S^{n}$.

We will show that the homotopy class of the clutching function $c: S^{n-1} \rightarrow \Omega_{1}^{n} S^{n}$ of the fibration $\tau_{n}^{+}$is exactly the boundary of $\iota_{n} \in \pi_{n}\left(S^{n}\right)$ in the long exact sequence associated to the evaluation fibration $\Omega_{1}^{n} S^{n} \rightarrow \Lambda_{1}^{n} S^{n} \rightarrow S^{n}$. The index 1 denotes the component of degree 1 maps. We identify $S^{n-1}$ to the equator of $S^{n}$. We take the north pole $N$ and the south pole $S$ respectively as base points in the domain and the range.

Let $H: I \times S^{n-1} \rightarrow S O(n+1) \rightarrow \Lambda_{1}^{n} S^{n}$ be the transformation such that $H(t, a)$ rotates by $t \pi$ the plane generated by $a$ and $N$, and fixes the orthogonal complement. The clutching function of $\tau_{n}^{+}$is $c=H\left(1,{ }_{-}\right): S^{n-1} \rightarrow \Omega_{1}^{n} S^{n}$. Let us identify the unreduced suspension of $S^{n-1}$ to $S^{n}$ via $[t, a] \mapsto H(t, a)(N)$. Then $H$ defines an element in $\pi_{n}\left(\Lambda_{1}^{n} S^{n}, \Omega_{1}^{n} S^{n}\right)$ projecting to the generator $\iota_{n} \in \pi_{n}\left(S^{n}\right)$, and $c=\partial \iota_{n}$. But by a theorem of Whitehead [12] $\partial\left(\iota_{n}\right)$ is adjoint to $\left[\iota_{n}, \iota_{n}\right]$, up to sign convention.

Let $w: S_{+}^{n-1} \wedge X \rightarrow \Omega^{n} \Sigma^{n} X$ be the composite of $c_{+} \wedge i: S_{+}^{n-1} \wedge X \rightarrow \Omega_{1}^{n} S_{+}^{n} \wedge \Omega^{n} \Sigma^{n} X$ and of the composition operation $o: \Omega_{1}^{n} S_{+}^{n} \wedge \Omega^{n} \Sigma^{n} X \rightarrow \Omega^{n} \Sigma^{n} X$ given by $o(\psi, f)=f \psi$.

We call the map $k(s, x, y)=\lambda(s, w(s, x), y)$ the twisted Browder map.

Theorem 11. There is a homotopy pushout

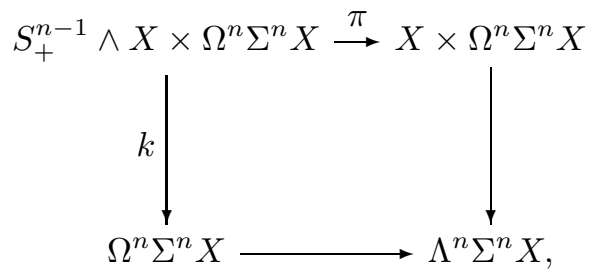

where $k$ is the twisted Browder map and $\pi$ is the projection.

Proof. We identify the two hemispheres to the unit disc by orientation preserving diffeomorphisms and we proceed as in Theorem [6] The main difference is that the trivializations regard the domain but not the range of the mapping space.

Thus the twisted Browder map $k$ replaces $\lambda$ in the proof, by comparing the trivializations of the one-point compactified tangent bundle on the two hemispheres with Lemma 10] 
In the next corollary $X$ is supposed to have finite type. Otherwise we must replace the expression 'cohomology algebra' by 'homology coalgebra'.

Corollary 12. The homology of $\Lambda^{n} \sum^{n} X$ with coefficients in a field depends only on the cohomology algebra of $X$ and is the direct sum of the suspended kernel and the cokernel of the twisted Browder action.

Proof. Let $\iota \in H_{0}\left(\Omega_{1}^{n} S^{n}\right)$ be the class of the identity map. By the diagram at p. 215 in 3 the Hurewicz homomorphism sends the adjoint Whitehead product in $\pi_{n-1}\left(\Omega_{2}^{n} S^{n}\right)$ to the Browder operation $[\iota, \iota] \in H_{n-1}\left(\Omega_{2}^{n} S^{n}\right)$, with our sign convention. Therefore, for $x \in H(X), w_{*}\left(e_{n-1} \otimes x\right)=$ $o_{*}\left([\iota, \iota] \iota^{-1} \otimes x\right)$ is the composition product in homology, where we identify $x$ to $i_{*}(x) \in H\left(\Omega^{n} \Sigma^{n} X\right)$. Suppose that the iterated coproduct $\Delta: H(X) \rightarrow H\left(X^{3}\right)$ is $\Delta(x)=\sum_{i} x_{i}^{\prime} \otimes x_{i}^{\prime \prime} \otimes x_{i}^{\prime \prime \prime}$.

Let $\chi$ be the conjugation of the Hopf algebra $H\left(\Omega^{n} \Sigma^{n} X\right)$. It depends only on the cohomology algebra of $X$, by induction and by Proposition 1.5 of $[3]$.

Then the twisted Browder action is $k_{*}(x \otimes y)=[x, y]+\sum_{i}\left[x_{i}^{\prime}, x_{i}^{\prime \prime}\right] \chi\left(x_{i}^{\prime \prime \prime}\right) y$.

Compare also Theorem 3.2 (iv) of [4].

\section{Examples and applications}

Corollary 13. At the prime 2 the homology of $\Lambda^{n} \Sigma^{n} X$ depends just on the homology of $X$, and is isomorphic to the homology of the section space $\Gamma\left(\tau_{n}^{+}(X)\right)$.

Proof. The Browder operation $[\iota, \iota]$ is trivial mod 2 .

Corollary 14. If $X$ is connected then $\Lambda^{n} \Sigma^{n} X$ and $\Gamma\left(\tau_{n}^{+}(X)\right)$ have the same rational homology.

Proof. Rationally any suspension is a wedge of spheres, so that $\Sigma^{n} X$ is rationally a $(n+1)$-fold suspension $\Sigma^{n+1} Y$, but $\Lambda^{n} \Sigma^{n+1} Y \simeq \Gamma\left(\tau_{n}^{+}(\Sigma Y)\right)$ has the same homology as $\Gamma\left(\tau_{n}^{+}(X)\right)$ by Corollary 7.

The corollary is not true in general, as the following example shows.

Example 15. The spaces $\Lambda^{2}\left(S^{2} \vee S^{2}\right)$ and $\Gamma\left(\tau_{2}^{+} \wedge\left(S^{0} \vee S^{0}\right)\right)$ have not the same rational homology componentwise.

Proof. Both spaces have components indexed by $\mathbb{Z} \times \mathbb{Z}$. The bifiltration of the section space $\Gamma=$ $\Gamma\left(\tau_{2}^{+} \wedge\left(S^{0} \vee S^{0}\right)\right)$ by fiberwise bidegree is compatible with the bifiltration, by number of particles, of the configuration space $C\left(S^{2}, S^{0} \vee S^{0}\right)$ of bicoloured particles on the sphere. Compare also Example [8] The bifiltration of the mapping space $M=\Lambda^{2}\left(S^{2} \vee S^{2}\right)$ is given by ordinary bidegree.

Let us denote the generators of $\tilde{H}_{0}\left(S^{0} \vee S^{0}\right) \subset \tilde{H}_{0}\left(\Omega^{2}\left(S^{2} \vee S^{2}\right)\right)$ by $x$ and $y$. It turns out from Corollary $\mathbf{7}$ that the homology group $H_{1}\left(\Gamma_{m, n}\right)$ is the quotient of

$$
H_{1}\left(\Omega_{m, n}^{2}\left(S^{2} \vee S^{2}\right)\right)=\mathbb{Q}\left\{[x, x] x^{m-2} y^{n},[x, y] x^{m-1} y^{n-1},[y, y] x^{m} y^{n-2}\right\}
$$

by the subspace generated by $\left[x, x^{m-1} y^{n}\right]$ and $\left[y, x^{m} y^{n-1}\right]$. But these elements have respectively coordinates $(m-1, n, 0)$ and $(0, m, n-1)$ with respect to the basis above. It follows that $H_{1}\left(\Gamma_{i, j}\right) \cong \mathbb{Q}^{2}$ for $(i, j)=(1,1)$ or $(i, j)=(0,1)$ or $(i, j)=(1,0)$ and $H_{1}\left(\Gamma_{i, j}\right) \cong \mathbb{Q}$ in all other cases.

On the other hand by Corollary $12 H_{1}\left(M_{m, n}\right)$ is the quotient of $H_{1}\left(\Omega_{m, n}^{2}\left(S^{n} \vee S^{n}\right)\right)$ by the subspace generated by $\left[x, x^{m-1} y^{n}\right]+[x, x] x^{m-2} y^{n}$ and $\left[y, x^{m} y^{n-1}\right]+[y, y] x^{m} y^{n-2}$, that have coordinates $(m, n, 0)$ and $(0, m, n)$. Thus $H_{1}\left(M_{0,0}\right) \cong \mathbb{Q}^{3}$ and $H_{1}\left(M_{i, j}\right) \cong \mathbb{Q}$ for any $(i, j) \neq(0,0)$. In particular $M_{0,0}$ differs in homology by all components of $\Gamma$.

The next example shows that for $n$ even and at odd primes the homology of the mapping space $\Lambda^{n} \sum^{n} X$ does not depend just on the additive homology of $X$, so that in particular the section space and the mapping space have not the same homology. 
Example 16. The spaces $X=S^{2} \vee S^{4} \vee S^{6}$ and $Y=\mathbb{C P}^{3}$ have the same additive homology, but $H_{9}\left(\Lambda^{2} \Sigma^{2} X ; \mathbb{Z}_{3}\right) \cong \mathbb{Z}_{3} \oplus \mathbb{Z}_{3}$ and $H_{9}\left(\Lambda^{2} \Sigma^{2} Y ; \mathbb{Z}_{3}\right) \cong \mathbb{Z}_{3}$.

Proof. Let use denote the homology generators of the homology of $X=S^{2} \vee S^{4} \vee S^{6}$ by $a_{1}, a_{2}, a_{3}$. We must compute the Browder action mod 3

$$
\lambda_{i}: \bigoplus_{j} \Sigma \tilde{H}_{j}(X) \otimes H_{i-1-j}\left(\Omega^{2} \Sigma^{2} X\right) \rightarrow H_{i}\left(\Omega^{2} \Sigma^{2} X\right)
$$

for $i=8,9$. For $i=8$ the left hand side is generated by $a_{1} \otimes\left[a_{1}, a_{1}\right]$ so that $\lambda_{8}=0$ by Theorem $1.2(6)$ in $\left[3\right.$. For $i=9$ the left hand side has dimension 6 , with generators $a_{1} \otimes a_{3}, a_{1} \otimes$ $a_{1}^{3}, a_{1} \otimes a_{1} a_{2}, a_{2} \otimes a_{2}, a_{2} \otimes a_{1}^{2}, a_{3} \otimes a_{1}$, and the right hand side has dimension 5 , with generators $\left[a_{1}, a_{3}\right],\left[a_{1}, a_{1}\right] a_{2},\left[a_{1}, a_{2}\right] a_{1},\left[a_{2}, a_{2}\right],\left[a_{1}, a_{1}\right] a_{1}^{2}$.

By the Poisson relation (Theorem $1.2(5)$ in $[3]$ ) $\left[a_{1}, a_{1}^{3}\right]=3\left[a_{1}, a_{1}\right] a_{1}^{2}=0 \bmod 3$. Thus the image of $\lambda_{9}$ has dimension 4 , with generators $\left[a_{1}, a_{3}\right],\left[a_{1}, a_{1}\right] a_{2}+\left[a_{1}, a_{2}\right] a_{1},\left[a_{2}, a_{2}\right], 2\left[a_{2}, a_{1}\right] a_{1}$, and $H_{9}\left(\Lambda^{2} \Sigma^{2}\left(S^{2} \vee S^{4} \vee S^{6}\right) ; \mathbb{Z}_{3}\right) \cong \operatorname{ker}\left(\lambda_{8}\right) \oplus \operatorname{coker}\left(\lambda_{9}\right) \cong \mathbb{Z}_{3} \oplus \mathbb{Z}_{3}$

Let us consider now the case of the projective space $\mathbb{C P}^{3}$, with homology generators $e_{1}, e_{2}, e_{3}$. We must check the twisted Browder action

$$
k_{i}: \bigoplus_{j} \Sigma \tilde{H}_{j}\left(\mathbb{C P}^{3}\right) \otimes H_{i-1-j}\left(\Omega^{2} \Sigma^{2} \mathbb{C P}^{3}\right) \rightarrow H_{i}\left(\Omega^{2} \Sigma^{2} \mathbb{C P}^{3}\right)
$$

for $i=8,9$. By the proof of Corollary 12$] k_{*}\left(\Sigma e_{1} \otimes y\right)=\left[e_{1}, y\right], k_{*}\left(\Sigma e_{2} \otimes y\right)=\left[e_{2}, y\right]+\left[e_{1}, e_{1}\right] y$ and

$$
k_{*}\left(\Sigma e_{3} \otimes y\right)=\left[e_{3}, y\right]+2\left[e_{1}, e_{2}\right] y-\left[e_{1}, e_{1}\right] e_{1} y .
$$

For the same reason as above $k_{8}=0$. Moreover the image of $k_{9}$ is generated by the five elements $\left[e_{1}, e_{3}\right],\left[e_{1}, e_{1}\right] e_{2}+\left[e_{1}, e_{2}\right] e_{1},\left[e_{1}, e_{1}\right] e_{2}+\left[e_{2}, e_{2}\right], 2\left[e_{1}, e_{2}\right] e_{1}+\left[e_{1}, e_{1}\right] e_{1}^{2},\left[e_{1}, e_{3}\right]+2\left[e_{1}, e_{2}\right] e_{1}-\left[e_{1}, e_{1}\right] e_{1}^{2}$.

Elementary linear algebra shows that they are linearly independent. Thus $k_{9}$ is surjective, and $H_{9}\left(\Lambda^{2} \Sigma^{2} \mathbb{C P}^{3} ; \mathbb{Z}_{3}\right) \cong \operatorname{ker}\left(k_{8}\right) \oplus \operatorname{coker}\left(k_{9}\right) \cong \mathbb{Z}_{3}$.

We compute next the homology of $\Lambda^{n} S^{n+k}$ for $k \geq 0$. We recall the computation of the homology of $\Omega^{n} S^{n+k}$ for $n>1$.

Let $X$ be a space acted on by the operad of little $n$-discs. For example $X=\Omega^{n} Y$ or $X=C\left(\mathbb{R}^{n}, Y\right)$. We use the lower index notation for Dyer-Lashof operations and do not consider $Q_{0}$ here.

a) Case $p=2$ :

There are operations $Q_{i}: H_{q}\left(X ; \mathbb{Z}_{2}\right) \rightarrow H_{2 q+i}\left(X ; \mathbb{Z}_{2}\right)$ for $0<i<n$. (These operation are also named $\xi_{i}$ in [3]). A string $Q_{I}$ of symbols $Q_{i}$ represents the composite operation. It is admissible if the sequence of indices is weakly monotone. The empty string is admissible and represents the identity.

b) Case $p$ odd:

There are operations $Q_{i}(x): H_{q}\left(X ; \mathbb{Z}_{p}\right) \rightarrow H_{p q+i(p-1)}\left(X ; \mathbb{Z}_{p}\right)$, defined for $0<i<n$ and when $i$ and $q$ have the same parity. Note that the operations $Q_{i}$ keep the parity and the Bockstein operator $\beta$ switches parity. An ordered string $Q_{I}$ of symbols $Q_{i}$ and $\beta$ represents the composite operation. We say that the operation $Q_{I}(x)$ is admissible if

1) the last symbol of the non-empty string $Q_{I}$ is $Q_{i}$, and $i$ has the same parity as the degree of $x$;

2 ) the sequence of indices is weakly monotone;

$3)$ the indices of two adjacent $Q^{\prime} s$ have the same parity;

4) the indices of two $Q^{\prime} s$ separated by a $\beta$ have opposite parity;

5) two adjacent $\beta$ do not appear.

In particular the empty string is admissible and represents the identity.

c) In characteristic 0 only the identity is admissible. 
Recall that $C\left(\mathbb{R}^{n}, S^{k}\right) \simeq \Omega^{n} S^{n+k}$ for $k>0$ 2. Let $\iota \in H_{k}\left(C\left(\mathbb{R}^{n}, S^{k}\right)\right)$ be the fundamental class of $S^{k} \simeq C_{1}\left(\mathbb{R}^{n}, S^{k}\right)$.

\section{Proposition 17. 3}

1) The homology of $C\left(\mathbb{R}^{n}, S^{k}\right)$ is the free commutative graded algebra on the admissible operations $Q_{I}(\iota)$, and in addition on the admissible operations $Q_{J}([\iota, \iota])$ if $n$ and $k$ have the same parity and $\operatorname{char}(K) \neq 2$.

2) The homology of $\Omega^{n} S^{n}$ is obtained from $C\left(\mathbb{R}^{n}\right)=C\left(\mathbb{R}^{n}, S^{0}\right)$ by inverting formally $\iota$.

3) The basis of $H\left(C\left(\mathbb{R}^{n}\right)\right)$ consisting of products of admissible operations has an integral degree $\nu$ that keeps track of the component, corresponding to the number of particles. This degree is generated by the rules $\nu(1)=0, \nu(\iota)=1, \nu([\iota, \iota])=2, \nu(x y)=\nu(x)+\nu(y)$ and $\nu\left(Q_{i}(x)\right)=\nu\left(\beta Q_{i}(x)\right)=p \nu(x)$.

Now we are ready to state the computation. The next theorem for $k>0$ gives also the homology of $\Lambda^{n} S^{n+k} \simeq C\left(S^{n}, S^{k}\right)$ by Proposition 9(1). Let $F$ denote the graded commutative algebra generated by a set of vectors, and let $Q$ be the set of all admissible operations on $\iota$ and $[\iota, \iota]$ except the identity.

\section{Theorem 18.}

1) For $n+k$ odd or for $\operatorname{char}(K)=2$

$$
H\left(C\left(S^{n}, S^{k}\right)\right) \cong H\left(C\left(\mathbb{R}^{n}, S^{k}\right)\right) \oplus \Sigma^{n+k} H\left(C\left(\mathbb{R}^{n}, S^{k}\right)\right) .
$$

2) For $n$ odd, $k$ odd and $\operatorname{char}(K) \neq 2$

$$
H\left(\Lambda^{n} S^{n+k}\right) \cong H\left(\Omega^{n} S^{n+k}\right) /([\iota, \iota]) \oplus \Sigma^{n+k} H\left(\Omega^{n} S^{n+k}\right) /(\iota) .
$$

3) For $n$ even, $k$ even and $p=\operatorname{char}(K)$ odd

$$
H\left(C\left(S^{n}, S^{k}\right)\right) \cong F(\iota, Q) \oplus \iota^{p-1}[\iota, \iota] F\left(\iota^{p}, Q\right) \oplus \Sigma^{n+k}[\iota, \iota] F(\iota, Q) \oplus \Sigma^{n+k} F\left(\iota^{p}, Q\right) .
$$

4) For $n$ even, $k$ even and $\operatorname{char}(K)=0$

$$
H\left(C\left(S^{n}, S^{k}\right)\right) \cong F(\iota) \oplus \Sigma^{n+k}[\iota, \iota] F(\iota) \oplus \Sigma^{n+k}\{1\} .
$$

5) In the case $k=0$ the component degree of $C\left(S^{n}\right)$ is obtained from the degree of $C\left(\mathbb{R}^{n}\right)$ by the additional rule $\nu\left(\Sigma^{n+k} x\right)=\nu(x)+1$.

Proof. We apply Corollary $\mathbf{7}$ The Browder action is given by $[\iota,-]$. In case 1$)$ the bracket $[\iota, \iota]$ is trivial. In all cases the bracket $[\iota, x]$ is trivial if $x$ is any generator of the algebra $H\left(\Omega^{n} S^{n+k}\right)$ other than $\iota$. Namely $[\iota,[\iota, \iota]]=0$ by $1.2(6)$ of $\left[\underline{3}\right.$, and consequently $\left[\iota, Q_{I}(\iota)\right]=0$ and $\left[\iota, Q_{J}([\iota, \iota])\right]=0$ by $1.2(8)$ and $1.3(9)$ of $[3]$. The action is a derivation with respect to the product by $1.2(5)$ of $[3]$. This completes the computation.

The computation of the homology of configuration spaces of even spheres at odd primes is not deducible from the methods of [1].

It remains to compute the homology of $\Lambda^{n} S^{n}$.

\section{Theorem 19.}

1) For $n$ odd or $\operatorname{char}(K)=2 \quad H\left(\Lambda^{n} S^{n}\right)=H\left(\Omega^{n} S^{n}\right) \otimes H\left(S^{n}\right)$.

2) For $n$ even and char $(K)=0 \quad H\left(\Lambda_{0}^{n} S^{n}\right)=H\left(S^{n-1}\right) \otimes H\left(S^{n}\right)$ and $H\left(\Lambda_{k}^{n} S^{n}\right) \cong H\left(S^{2 n-1}\right)$ for $k \neq 0$.

3) For $n$ even and char $(K)=p$ odd $H\left(\Lambda_{k}^{n} S^{n}\right) \cong H\left(\Omega_{0}^{n} S^{n}\right) \otimes H\left(S^{n}\right)$ if $p$ divides $k$ and $H\left(\Lambda_{k}^{n} S^{n}\right) \cong F(Q) \oplus \Sigma^{n}[\iota, \iota] F(Q)$ if $(p, k)=1$. 
Proof. Since the bundle $\tau_{n}^{+}$is trivial, there is a homeomorphism $\Gamma\left(\tau_{n}^{+}\right) \cong \Lambda^{n} S^{n}$, and we can use either Corollary 7 or Corollary 12 For $n$ odd both the untwisted and the twisted Browder actions are trivial. For $n$ even note that the base point of $\Gamma\left(\tau_{n}^{+}\right)$, the section at infinity, is the antipodal map of $S^{n}$, that has degree -1. If $\Gamma_{k}$ is the component of $\Gamma\left(\tau_{n}^{+}\right)$of fiberwise degree $k$, then $\Gamma_{k+1} \cong$ $\Lambda_{k}^{n} S^{n}$. This is compatible with corollaries 7 and 12. the Browder action $x \mapsto[\iota, x]$ from $\Omega_{k}^{n} S^{n}$ to $\Omega_{k+1}^{n} S^{n}$ computes the homology of $\Gamma_{k+1}$, but the twisted Browder action from $\Omega_{k-1}^{n} S^{n}$ to $\Omega_{k}^{n} S^{n}$ $\tau(x)=[\iota, x]+[\iota, \iota] \iota^{-1} x$ computes the homology of $\Lambda_{k}^{n} S^{n}$. They are compatible because $\iota \tau(x)=$ $[\iota, x] \iota+[\iota, \iota] x=[\iota, \iota x]$. Cases 1) and 2) are easy. In case 3) the multiplication by $\iota^{p}$ induces an isomorphism $H\left(\Lambda_{k}^{n} S^{n}\right) \cong H\left(\Lambda_{k+p}^{n} S^{n}\right)$. If $(p, k)=1$ then the composition by a degree $k$ map induces an isomorphism $H\left(\Lambda_{1}^{n} S^{n}\right) \cong H\left(\Lambda_{k}^{n} S^{n}\right)$. One concludes the computation by keeping track of the component degree.

\section{Homotopy pullbacks}

Another approach to our spaces is given by homotopy pullbacks, by decomposing the sphere $S^{n}$, basis of the evaluation fibration, as union of two $n$-discs.

Proposition 20. The mapping space is the homotopy pullback

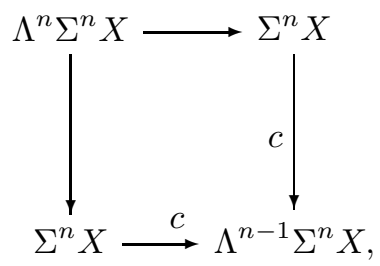

where $c$ is adjoint to the projection $S_{+}^{n-1} \wedge \Sigma^{n} X \rightarrow \Sigma^{n} X$.

Proposition 21. The section space $\Gamma\left(\tau_{n}^{+}(X)\right)$ is the homotopy pullback

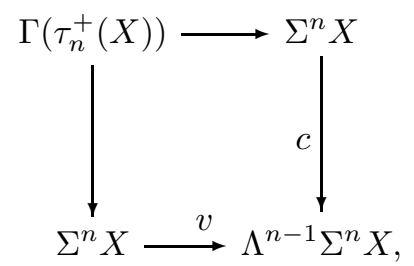

where $v: \Sigma^{n} X \rightarrow \Lambda^{n-1} \Sigma^{n} X$ is adjoint to $w: S_{+}^{n-1} \wedge X \rightarrow \Omega^{n} \Sigma^{n} X$.

The section and mapping spaces are both total spaces of fibrations with same fiber and basis. The mapping space evaluation fibration admits the section given by constant loops. Therefore for $i>0$ $\pi_{i}\left(\Lambda^{n} \Sigma^{n} X\right)=\pi_{i+n}\left(\Sigma^{n} X\right) \oplus \pi_{i}\left(\Sigma^{n} X\right)$. The section space fibration admits just a stable section, given by the inclusion $S_{+}^{n} \wedge X=C_{1}\left(S^{n}, X\right) \rightarrow \Gamma\left(\tau_{n}^{+}(X)\right)$, since $\Sigma\left(S_{+}^{n} \wedge X\right) \simeq \Sigma\left(\Sigma^{n} X \vee X\right)$.

Example 22. $C\left(S^{2}, \mathbb{C P}^{2}\right)$ and $\Lambda^{2} \Sigma^{2} \mathbb{C P}^{2}$ are not rationally homotopy equivalent, but they have the same homology over any field.

Proof. We show first that the $\pi_{5}^{\prime} s$ of the two spaces have different ranks. Rationally $\Sigma^{2} \mathbb{C P}^{2} \simeq S^{4} \vee S^{6}$, so that $r k\left(\pi_{5}\left(\Lambda^{2} \Sigma^{2} \mathbb{C P}^{2}\right)\right)=r k\left(\pi_{7}\left(S^{4} \vee S^{6}\right)\right)+r k\left(\pi_{5}\left(S^{4} \vee S^{6}\right)\right)=1$.

The fiber square of Proposition 21] induces a long sequence of rational homotopy groups

$$
\ldots \pi_{i}\left(C\left(S^{2}, \mathbb{C P}^{2}\right)\right) \longrightarrow \pi_{i}\left(\Sigma^{2} \mathbb{C P}^{2}\right) \oplus \pi_{i}\left(\Sigma^{2} \mathbb{C P}^{2}\right) \stackrel{c_{\#} \oplus v_{\#}}{\longrightarrow} \pi_{i}\left(\Lambda \Sigma^{2} \mathbb{C P}^{2}\right) \longrightarrow \pi_{i-1}\left(C\left(S^{2}, \mathbb{C P}^{2}\right)\right) \ldots
$$

Now $\pi_{5}\left(S^{4} \vee S^{6}\right)$ has rank 0 , so that rationally $\pi_{5}\left(C\left(S^{2}, \mathbb{C P}^{2}\right)\right)$ is the quotient of $\pi_{6}\left(\Lambda \Sigma^{2} \mathbb{C P}^{2}\right)=$ $\mathbb{Q}\{s, u\}$ by the image of $c_{\#} \oplus v_{\#}$, defined on $\pi_{6}\left(\Sigma^{2} \mathbb{C P}\right)^{2}=\mathbb{Q}\left\{s_{1}, s_{2}\right\}$. 
Here $s_{1}$ and $s_{2}$ represent the generators coming from the wedge summand $S^{6} \rightarrow S^{4} \vee S^{6}$ and $s=c_{\#}\left(s_{1}\right)=c_{\#}\left(s_{2}\right)$. Moreover $u$ is the composition $S^{6} \rightarrow \Omega S^{4} \rightarrow \Lambda S^{4} \rightarrow \Lambda\left(S^{4} \vee S^{6}\right)$, with the first map adjoint to the Whitehead product $\left[\iota_{4}, \iota_{4}\right]: S^{7} \rightarrow S^{4}$.

The group $\left[S_{+}^{1} \wedge \mathbb{C P}^{2}, \Omega^{2} \Sigma^{2} \mathbb{C P}^{2}\right]$ splits as $\left[\Sigma \mathbb{C P}^{2}, \Omega^{2} \Sigma^{2} \mathbb{C P}^{2}\right] \oplus\left[\mathbb{C P}^{2}, \Omega^{2} \Sigma^{2} \mathbb{C P}^{2}\right]$, because after two suspensions $S_{+}^{1}$ and $S^{1} \vee S^{0}$ are equivalent as co-H spaces. Rationally the first summand $\pi_{5}\left(\Omega^{2} \Sigma^{2} \mathbb{C P}^{2}\right)=\mathbb{Q}\{\bar{u}\}$ has rank 1 and is detected by the action on $\pi_{5} \cong H_{5}$.

But $w: S_{+}^{1} \wedge \mathbb{C P}^{2} \rightarrow \Omega^{2} \Sigma^{2} \mathbb{C P}^{2}$ induces the nontrivial action $w_{*}\left(\left[S^{1}\right] \otimes e_{2}\right)=\left[e_{1}, e_{1}\right]$ where $e_{i} \in H_{2 i}\left(\mathbb{C P}^{2}\right)$ is the generator, by the proof of Corollary [12] Thus $w$ corresponds in the decomposition above to $\bar{u} \oplus i$, with $i$ adjoint to the identity. By taking adjoints, $v: \Sigma^{2} \mathbb{C P}^{2} \rightarrow \Lambda \Sigma^{2} \mathbb{C P}^{2}$ represents the sum in the group $\left[\Sigma^{2} \mathbb{C P}^{2}, \Lambda \Sigma^{2} \mathbb{C P}^{2}\right]$ of the injection $c: \Sigma^{2} \mathbb{C P}^{2} \rightarrow \Lambda \Sigma^{2} \mathbb{C P}^{2}$ and the composition $\Sigma^{2} \mathbb{C P}^{2} \rightarrow S^{6} \stackrel{u}{\longrightarrow} \Lambda \Sigma^{2} \mathbb{C P}^{2}$.

It follows that $v_{\#}\left(s_{2}\right)=s+u$, and $\pi_{5}\left(C\left(S^{2}, \mathbb{C P}^{2}\right)\right)$ has rank 0 .

The second assertion follows from Corollary 13 and the fact that away from the prime $2 \Sigma^{2} \mathbb{C P}^{2} \simeq$ $S^{4} \vee S^{6}$.

This shows that the rational homotopy type of $C\left(S^{2}, X\right)$ does not depend just on the rational homology of $X$, by taking $X=\mathbb{C P}^{2}$ and $X=S^{2} \vee S^{4}$.

Dipartimento di Matematica, Università di Roma "Tor Vergata", Via della Ricerca Scientifica 1, 00133 Roma, Italy

e-mail:salvator@mat.uniroma2.it

\section{References}

[1] C.-F. Boedigheimer, F.R. Cohen, L. Taylor, Homology of configuration spaces, Topology 28 (1989), 111-123.

[2] C.-F. Boedigheimer, Stable splittings of mapping spaces, LNM 1286, 1987, 174-187.

[3] F.R. Cohen, The homology of $C_{n+1}$-spaces, $n \geq 0$, LNM 533, 1976, 207-351.

[4] F.R. Cohen, The homology of $S F(n+1)$, LNM 533, 1976, 352-398.

[5] W.S. Massey, The homotopy type of certain configuration spaces, Bol.Soc.Mat.Mex. 37 (1992), 355-365.

[6] J.P. May, The geometry of iterated loop spaces, LNM 271, Springer, 1972.

[7] D. McDuff, Configuration spaces of positive and negative particles, Topology 14 (1975), 91-107.

[8] L. Menichi, The cohomology ring of free loop spaces, Homology Homotopy Appl. 3 (1) (2001), 193-224.

[9] R.J. Milgram, Iterated loop spaces, Ann. Math. 84 (1966), 386-403.

[10] T. Pirashvili, Hodge decomposition for higher order Hochschild homology, Ann. Ec. Norm. Sup. (4) 33 (2) (2000), 151-179.

[11] P. Salvatore and N. Wahl, Framed discs operads and Batalin-Vilkovisky algebras, to appear in Quart. J. Math.

[12] G.W. Whitehead, On products in homotopy groups, Ann. of Math. 47 (1946), 460-475. 